\title{
Analyse de réseaux et histoire
}

\author{
Claire LEMERCIER
}

Le mot « réseau » est partout aujourd'hui ${ }^{1}$, y compris en histoire : " réseaux de famille, d'alliance, de parenté, de proximité, de voisinage, entourage, espace de connivence, chaînes de connaissances, réseaux de fidélités, d'amitié, de clientèle, de sociabilité, de pouvoirs, de crédits, d'origine, réseaux marchands, intellectuels, professionnels, épistolaires, diplomatiques, religieux, maçonniques, migratoires, clandestins, latents $\|^{2} \ldots$ Malgré des appels récurrents ${ }^{3}$, l'usage métaphorique du terme s'avère difficile à dépasser. Cela dit, l'intérêt pour les réseaux en sciences sociales ne renvoie pas seulement à une mode : il est lié à la volonté de dépasser déterminisme des structures et modèle simpliste du choix rationnel, en s'intéressant au lien social, aux aspects organisationnels, à ce que certains appellent le niveau "méso ${ }^{4}$. Mais on ne saurait se contenter de repérer l'existence de telle ou telle forme de "réseau ", car il faut surtout se demander si ces "réseaux" sont ou non hiérarchisés, multipolaires, mouvants ou tenaces, à quels moments ils fonctionnent plutôt comme des ressources ou au contraire comme des contraintes pour les individus ou les groupes...Autant de questionnements qui permettent d'éviter les plus simplistes théories de la modernité, pour lesquelles le "réseau » est quelque chose d'archaïque (regretté pour sa chaleur perdue ou dénoncé pour son poids persistant), ou

1. Cet article n'existerait pas sans Noël Bonneuil, Alain Chatriot, Marie Chessel, Pierre François, Emmanuel Lazega, Hélène Lemesle, Lise Mounier et Paul-André Rosental. Je remercie également les rédacteurs et le secrétariat de la $R H M C$, ainsi que les participants de divers séminaires où j'ai pré-senté des versions préliminaires, à I'EHESS, à I'ENS Cachan, à la MMSH d'Aix-en-Provence et à I'IHMC, en particulier Jean Boutier, Wolfgang Kaiser et Antoine Lilti.

2. Selon le diagnostic de Jean BOUTIER, "Sources, objets, outils. Quelques remarques pour éviter de conclure ", in Pierre-Yves Beaurepaire, Dominique TaURISSON (éd.), Les ego-documents à l'heure de l'électronique. Nouvelles approches des espaces et des réseaux relationnels, Montpellier, Presses universitaires de Montpellier, 2003, p. 535-544 (citation p. 539) et www.egodoc.revues.org/octobre2002/.

3. Notamment, en français, Jean-Pierre DeDIEU, Zacarías MoutouKIAS, «Introduction. Approche de la théorie des réseaux sociaux", in Juan Luis CASTellano, Jean-Pierre Dedieu (dir.), Réseaux, familles et pouvoirs dans le monde ibérique à la fin de l'Ancien Régime, Paris, CNRS Éditions, 1998, p. 7-30.

4. Paul-André Rosental, "Pour une analyse mésoscopique des migrations», Annales de démographie historique, 2002, $\mathrm{n}^{\circ}$ 2, p. 145-160; Emmanuel LAZEGA, "Rationalité, discipline sociale et struc-ture ", Revue française de sociologie, 44, 2003, p. 305-329. 
bien est le summum de la modernité, avec "l'organisation en réseau» des managers, horizontale et souple, opposée à la bureaucratie ${ }^{5}$.

Bref, ne pas simplement chercher si un réseau existe ou s'il a remplacé l'institution ou la classe, mais prendre au sérieux la notion, étudier la configuration produite par divers types de liens, son évolution, ses effets : cette volonté conduit un nombre croissant d'historiens à s'intéresser à l'outil appelé "analyse de réseaux ", parfois avec des excès de prudence ou d'espoir, comme il est normal face à une technique récente. Le présent article se propose de pointer quelques apports de cet outil, quelques possibilités d'en user directement ou de s'en inspirer pour spécifier des concepts permettant de dépasser l'ambiguïté du mot "réseau». Il sera bien question d'un outil, complémentaire d'autres techniques quantifiées et des analyses qualitatives, et non d'un paradigme qui balaierait tout sur son passage. Il ne s'agit pas pour autant de remplacer les manuels ${ }^{6}$ et séminaires qui permettent de se former pour mettre en œuvre concrètement de telles analyses - ce qui, sans être très difficile, requiert un peu de temps ${ }^{7}$. Le but est de présenter un large éventail d'articles et livres ayant appliqué une analyse de réseaux un tant soit peu formalisée à des données historiques. Cette approche a donc ses limites: on n'évoquera pas les travaux, nombreux et riches néanmoins, qui n'ont pas recours à ce type de formalisation $^{8}$, ni ceux qui ne portent que sur des données sociologiques contemporaines; les travaux anglo- et francophones seront privilégiés, même s'il en existe dans d'autres langues. L'exhaustivité est de toute façon impossible, s'agissant d'un domaine en pleine expansion' ${ }^{9}$ et en même temps caractérisé par la dispersion des recherches. Ce sont en effet des individus ou des petits groupes isolés, pour l'essentiel de sociologues, qui se sont jusqu'ici penchés sur des sources historiques - certains ne voyant dans les archives qu'une source commode de données longitudinales, d'autres collaborant avec des

5. Sur cet usage du mot, cf. Luc Boltanski, Ève Chiapello, Le nouvel esprit du capitalisme, Paris, Gallimard, 2000.

6. Pierre Mercklé, Sociologie des réseaux sociaux, La Découverte, 2004 (simple et relativement critique); Alain Degenne, Michel Forsé, Les réseaux sociaux [1994], Paris, Armand Colin, 2004; Vincent Lemieux, Mathieu OuImet, L'analyse structurale des réseaux sociaux, Québec, Presses de l'Université Laval-Bruxelles, De Boeck Université, 2004 (plus techniques); V. Lemieux, À quoi servent les réseaux sociaux?, Sainte-Foy, Éditions de l'IQRC, 2000 (très peu technique); E. LAZEGA, Réseaux sociaux et structures relationnelles, Paris, PUF, 1998 (plus centré sur la sociologie des organisations et la sociologie économique).

7. Si les logiciels sont variés, on peut citer le plus utilisé, Ucinet (Stephen P. BorGatTi, Martin G. Everett, Linton C. Freeman, Ucinet 6 for Windows: Software for Social Network Analysis, Harvard, Analytic Technologies, 2002), et Pajek (de Vladimir Batagelj et Andrej Mrvar), puissant mais nécessitant un plus long apprentissage. Pour les logiciels, plans de cours, annonces d'événements, listes de discussion, voir le site www.insna.org.

8. Ce qui ne signifie pas qu'ils sont sans rigueur; mais il ne pouvait être question de mentionner toute la micro-histoire, les travaux sur les clientèles, les sociabilités, les groupes d'intérêt, etc.

9. Dont peut témoigner la comparaison avec les surveys de Mustafa EMIRBAYER, Jeff GoodwIN, "Network analysis, culture, and the problem of agency", American fournal of Sociology, 99/6, mai 1994, p. 1411-1454 (plus critique) et Bonnie H. ERICKSON, "Social networks and history. A review essay", Historical Methods, 30/3, été 1997, p. 149-157 (plus pédagogique). 
historiens, voire réalisant une véritable synthèse des disciplines. Que ce soit pour les critiquer ou pour s'en inspirer, il est utile de repérer au moins une partie de ces travaux, portant sur des terrains variés et trop souvent absents des bibliothèques françaises.

Après avoir défini, à partir d'un exemple, quelques grands concepts d'analyse de réseaux, on verra que cette technique n'a aujourd'hui produit que des résultats limités, en particulier en raison de problèmes de sources, en histoire sociale (famille, stratifications, élites...). En revanche, elle apparaît utile, de façon instrumentale, dans des domaines ou des disciplines peut-être plus inattendus comme la géographie historique, l'histoire des savoirs ou celle des entreprises. Le champ de recherche le plus dynamique se situe toutefois du côté du politique, de l'étude de l'engagement et des réseaux d'organisations.

\section{Quelques NOTIONS UTILES À EMPRUNTER À L'ANALYSE de RÉSEAUX}

Pourquoi s'intéresser à l'analyse de réseaux, si l'on n'est pas sûr de disposer de sources se prêtant à un tel traitement? Face à la confusion actuelle du vocabulaire, il semble intéressant de recourir à un certain nombre de concepts éprouvés, qui permettent de décrire, à différentes échelles, la morphologie d'un "réseau» : ainsi, même si l'usage en reste purement heuristique, du moins seront-ils clairement définis. Se confronter à l'analyse de réseaux amène en particulier à donner toute leur place aux questions de frontière ou de hiérarchie: ce n'est pas parce qu'il y a "du réseau» qu'il n'y a qu'un (ou des) groupe(s) uni(s) et solidaire(s), sans domination, incompréhensions, contrôle d'un seul sur les échanges ou rupture. Autant d'éléments qu'il est possible de décrire précisément, voire de mesurer.

\section{Le réseau et la toile}

À cet égard, le refus de nombreux chercheurs qui, par ailleurs, analysent finement différents liens sociaux de recourir à toute technique un tant soit peu quantifiée, d'utiliser les concepts de «l'analyse structurale» - ou bien leur choix de s'en tenir à des graphes en toile d'araignée, même lorsque la densité des liens les rend illisibles - apparaît indûment prudent, voire contre-productif ${ }^{10}$. Si la quantification ou l'usage de concepts standardisés n'ont pas vocation à remplacer la critique des sources ou la narration, ils offrent un minimum de comparabilité et rendent possible une discussion argumentée des résultats. En outre, le

10. Maurizio GriBaud, Alain Blum, «Des catégories aux liens individuels. L'analyse statistique de l'espace social", Annales ESC, 45/6, nov.-déc. 1990, p. 1365-1402; M. GriBaudi, "Les discontinuités du social. Un modèle configurationnel", in Bernard LEPETIT (dir.), Les formes de l'expérience. Une autre histoire sociale, Paris, Albin Michel, 1995, p. 187-225; Z. Moutoukias, "Réseaux de négociants ou réseaux ego centrés: une approche méthodologique", in P.-Y. BEAUREPAIRE, D. TAURISSON (éd.), Les ego-documents..., op. cit., p. 447-468. 
choix exclusif d'une représentation graphique peut avoir l'effet pervers, pour des lecteurs peu habitués à la lecture de graphes complexes (et qui y est réellement habitué ?), de donner une image d'objectivisme, de "cartographie du social». Or rien n'est moins cartographiable qu'un réseau: seuls les traits qui lient les points y sont définis, leur longueur et leur position sur le papier restant arbitraires, au choix du chercheur ou du logiciel. De plus, le graphe montre les liens bien plus que les non-liens (un réseau très peu dense peut déjà donner un graphe embrouillé), la réciprocité bien plus que l'échange inégal (même si on y ajoute des flèches), donc peut induire une image irénique du social, souvent réfutée par une analyse quantifiée des mêmes données. Utile pour représenter des réseaux petits ou peu denses, la représentation graphique n'est en rien, en elle-même, une méthode d'analyse.

Si le graphe n'est pas la panacée, que peut-on donc faire? À condition d'avoir défini précisément le(s) lien(s) et la population à étudier, il suffit de fournir au logiciel une liste de liens (sans formatage complexe) pour obtenir, selon le type de sources, différents indicateurs. Si l'on s'intéresse de façon indépendante aux relations entourant des individus, sans relier ces individuscentres entre eux (comme on le ferait à partir de questionnaires issus d'un sondage), on parle d'étude de "réseaux égocentrés»; si l'on dispose d'une information suffisamment exhaustive sur toute une population dont on étudie les liens internes de façon homogène, on parle d'"analyse structurale", et les indicateurs disponibles diffèrent. Pour les présenter, prenons l'exemple des "enquêtes collectives" favorisées par le CNRS dans la recherche en histoire. On peut considérer que le fait de participer ensemble à une enquête crée un lien entre deux individus. Indiqué dans un projet ou un rapport, ce lien recouvre probablement des situations différentes; mais on peut considérer que déclarer un travail commun, même si l'interaction réelle est faible, veut dire quelque chose: c'est donc en partie la rhétorique du rapport que l'on analyse. En particulier, comparer les liens déclarés à différentes périodes nous renseigne sur des évolutions qui relèvent à la fois de l'historiographie et de la division du travail scientifique ${ }^{11}$.

\section{Réseaux égocentrés et capital relationnel}

Dans notre exemple, on pourrait choisir un échantillon de directeurs de recherche, chargés de recherche et ingénieurs dans différents laboratoires et comparer, selon le grade, le sexe, l'ancienneté, le lieu d'exercice... le nombre de personnes avec lesquelles ils déclarent participer à des enquêtes et les attributs de ces personnes (grade, sexe, etc.). Taille et composition du réseau personnel

11. L'exemple donné ici est inspiré de C. LEMERCIER, «Le Centre de Recherches Historiques comme "structure fédérative" ? Réseaux de collaboration et thèmes de recherche (1974-1995)", colloque international de novembre 1999 sur «La recherche collective, une révolution des sciences sociales au $\mathrm{xx}^{\mathrm{e}}$ siècle?». 
sont les données de base que l'analyse égocentrée permet de comparer - à condition que les sources soient bien comparables. Si l'information existe, on peut aussi prendre en compte la corrélation entre types de liens - la question de la «multiplexité» des liens, en termes de réseaux: les personnes avec qui l'on mène des enquêtes collectives sont-elles aussi, par exemple, d'anciens doctorants du même directeur? Entretient-on avec elles des liens syndicaux, amicaux, etc. ? Et, ce qui est souvent encore plus difficile à savoir, sont-elles aussi liées entre elles? Dans le cas de la recherche collective, il est ainsi intéressant de savoir si une personne est insérée dans un petit groupe dense de recherche ou bien dans plusieurs qui par ailleurs s'ignorent (du fait par exemple qu'elle apporte une expertise technique ou linguistique). L'étude de réseaux égocentrés permet ainsi de comparer le "capital relationnel» de plusieurs individus (ou d'un même individu à plusieurs dates) suivant différentes dimensions: taille, composition en termes d'attributs, multiplexité ou cloisonnement des différents types de liens, densité (un réseau personnel est dense si ses membres sont par ailleurs interconnectés). En revanche, ce type d'étude ne permet pas de tenir un discours global sur la structure relationnelle de la population observée, puisqu'elle s'en tient à observer une collection d'individus assortis chacun de leur réseau.

\section{L'analyse structurale de réseaux: centralité, cliques, positions}

Cette deuxième approche s'applique à des données d'un type différent. Elle suppose de connaître les relations, ou l'absence de relation, entre chaque couple possible d'individus d'un ensemble donné. Il ne s'agit pas forcément de connaître tous les types de liens possibles, mais de viser l'exhaustivité sur un ou quelques types de liens. Cela nécessite une réflexion préalable d'une part sur la définition des liens à prendre en compte, d'autre part sur les frontières de la population à étudier. En effet, ses relations avec le reste du monde ne seront pas justiciables de l'analyse structurale formalisée, qui ne traite que des liens internes. Deux stratégies sont possibles: s'intéresser à une population prédéfinie par un critère extérieur, par exemple statutaire (ici, les membres du CRH, Centre de Recherches Historiques de l'École des Hautes Études en Sciences Sociales), ou bien partir d'un noyau d'individus proches et ajouter, pendant un certain nombre d'étapes, tous ceux à qui ils sont liés (par exemple les participants aux enquêtes extérieurs au $\mathrm{CRH}$, dont il faudrait alors connaître les liens entre eux). L'analyse structurale permet de lier les niveaux d'analyse "micro", "méso" et "macro». Elle peut en effet fournir des indicateurs décrivant la position de chaque individu dans le réseau étudié, des données sur les groupes "cohésifs" qui peuvent s'y distinguer et un résumé de la structure d'ensemble (frontières internes, hiérarchies...).

Au niveau individuel, de nombreux indicateurs de la "centralité sont disponibles, en lien avec différentes conceptions du "capital social». La définition la plus simple consiste à compter le nombre de liens d'un individu, 
éventuellement en distinguant leur sens et leur intensité : par exemple, dans le cas des enquêtes, on peut compter à combien de personnes chacun est relié, en tenant compte ou non des liens multiples avec la même personne; on peut aussi rendre le lien asymétrique en considérant non plus une collaboration égalitaire, mais un lien de chaque collaborateur au directeur de l'enquête. Mais, bien souvent, compter le nombre de liens ne suffit pas. On peut avoir envie, par exemple, de distinguer des personnes liées à des individus euxmêmes centraux: les logiciels d'analyse de réseaux calculent différentes variantes de ce type d'indicateur, qui permet d'éliminer les personnes disposant de nombreux liens, mais au sein d'une "niche" à l'écart du reste du réseau. Un dernier concept, celui d'«intermédiarité», définit la position du broker, celui qui représente le seul lien, le passage obligé, entre deux groupes qui, par ailleurs, s'ignorent - ce qui constitue, dans certaines situations, une position de pouvoir (par exemple par le biais du contrôle de l'information), dans d'autres, une position vulnérable. La morphologie seule, en effet, ne dit rien, mais, interprétée en fonction de la connaissance du terrain, elle peut être pleine d'enseignements.

Ainsi, à l'intérieur du CRH, en 1974-1978, les trois personnes ayant le plus de liens avec d'autres membres du Centre par le biais d'enquêtes collectives sont un chef de travaux et deux techniciennes contractuelles, également inscrits en thèse de $3^{\mathrm{e}}$ cycle et dont les compétences sont manifestement considérées comme généralistes. Savoir cela nous en dit beaucoup sur les modes de travail de l'époque; on peut dire que ces personnes sont "centrales", mais ont-elles du pouvoir, de l'influence? En revanche, les trois personnes les plus "intermédiaires» dans les enquêtes sont le directeur du laboratoire et deux autres membres d'instances administratives, alors maîtres-assistants. Leur intermédiarité provient de leur association à des enquêtes de méthodologie ou d'histoire de l'histoire: on peut penser qu'elles structurent le laboratoire, centralité administrative et scientifique se renforçant mutuellement. Ces phénomènes seraient restés inaperçus sans l'usage de l'analyse de réseaux, car le grand nombre d'enquêtes rend la structure incompréhensible à l'œil nu.

Si l'on passe des individus aux groupes, puis à l'allure générale du réseau étudié, on peut mettre en évidence des "cliques", des groupes "cohésifs" ou non, placés au centre ou à la périphérie du réseau. Les "cliques» sont des groupes à l'intérieur desquels tous les liens possibles sont réalisés. Les logiciels permettent de nuancer cette définition, mais l'enjeu est bien de repérer des groupes plus densément connectés que la moyenne - ce qui peut, selon les terrains, impliquer de la solidarité, du contrôle social ou de la redondance d'information. Dans notre exemple, on peut s'intéresser aux cliques si l'on quitte le point de vue des individus pour celui des enquêtes, en décidant par exemple que deux enquêtes sont liées si elles partagent au moins un membre. Dans ce cas, le repérage de cliques (au moins trois enquêtes reliées deux à deux entre elles) identifie des champs de recherche irrigués par plusieurs projets impliquant en partie les mêmes personnes. Il peut être intéressant de 
savoir si ces cliques se créent plutôt autour de méthodes (archéologie, informatique... comme c'est le cas en 1974-1978), de terrains géographiques ou de périodes historiques.

Enfin, les logiciels permettent de donner une vision globale de la structure du réseau, en termes de centre(s) et de périphérie(s), ou bien de leur absence si tout est homogène et décentralisé. Ce qui est ainsi produit, c'est un classement des individus étudiés (personnes ou enquêtes) selon leur "position» dans le réseau - étant entendu que c'est le chercheur qui décide du nombre de positions à prendre en compte et plus généralement qui contrôle tous les paramètres des analyses, ce qui évite les phénomènes de "boîte noire" et oblige à réfléchir à une stratégie de traitement des données. En termes techniques, on parle d' "équivalence» ("structurale» ou "régulière») pour évoquer ce qui fonde ce classement en "positions» (centre, périphérie, îlot isolé...): elles sont dites "équivalentes" par rapport à la structure d'ensemble. Ainsi, au sein du CRH en 1978-1980, on retrouve des groupes d'individus et d'enquêtes cohésifs et fermés sur eux-mêmes (en histoire médiévale), d'autres à la fois cohésifs en interne et très centraux, très reliés aux autres (en archéologie) et des "positions" périphériques, comprenant des enquêtes pas ou peu reliées, ni entre elles ni aux autres (ce qui veut souvent dire que leurs liens sont extérieurs au laboratoire). Cette technique est particulièrement utile pour démontrer l'existence de fractures au sein d'un réseau ou pour y définir une hiérarchie. Elle donne de plus une représentation simple de réseaux complexes, utilisable pour des comparaisons, dans l'espace ou dans le temps.

Les outils proposés par l'analyse de réseaux permettent donc de produire des indicateurs chiffrés et des schémas de synthèse fondés sur autre chose que l'intuition; en outre, même quand on ne les utilise pas directement, ils aident à réfléchir sur des concepts comme la centralité ou la cohésion. C'est l'usage de ces concepts qui fait entrer dans l'analyse de réseaux, plus que celui des logiciels, possible ou non selon les sources. En effet, une analyse formelle nécessite un certain degré d'exhaustivité et de comparabilité (ce qui implique de bien définir le lien observé); en outre, elle est plus riche si l'on dispose d'informations sur plusieurs types de liens, sur l'orientation et l'intensité de chaque lien et sur sa date ${ }^{12}$. Malgré ces exigences, des travaux finalement nombreux ont déjà appliqué des analyses de réseaux plus ou moins élaborées à des sources historiques relevant de périodes, terrains et sous-disciplines extrêmement variés.

12. La prise en compte de la temporalité dans les réseaux reste un problème central depuis les origines. Pour une proposition de formalisation (exigeante en termes de sources) de leur dynamique, voir le logiciel Siena de Tom Snijders (http://stat.gamma.rug.nl/snijders/siena.html). En pratique, c'est en général la narration qui reprend ses droits quand il s'agit de comprendre le passage d'un état du réseau à un autre. 


\section{RéseauX et hISTOIRE SOCIALE: SOCIABiLItÉs, FAMILLES, COMMUNAUTÉS}

Lorsque l'on pense "réseaux", ce sont souvent les liens "forts", construisant des communautés, qui viennent à l'esprit: liens familiaux, amicaux, de voisinage... objets d'une historiographie dynamique, qui n'a pas forcément besoin d'un outil plus formalisé. En effet, le problème des sources se pose de façon aiguë et paradoxale sur ces thèmes: leur richesse impose en général une étude plutôt qualitative, au détriment de la comparabilité et donc de la formalisation. Cependant, un dialogue avec des approches plus formelles peut s'avérer intéressant, notamment pour "dénaturaliser» les représentations, y compris graphiques, de la famille ${ }^{13}$.

\section{Famille, amitié... des sources non formalisables?}

L'étude des journaux intimes et des correspondances représente un courant historiographique bien vivant, qu'il s'agisse d'histoire de la famille, des sociabilités, d'histoire intellectuelle ou d'une histoire économique qui ne se coupe pas du social. Les tentatives de formalisation dans ce domaine ont été plus rares, bien que précoces. Alan MacFarlane, dans un journal intime du XVII ${ }^{\mathrm{e}}$ siècle, comptait ainsi les visites de différents types, évaluait les places respectives de la famille, de la belle-famille et des voisins, les situait sur des cartes, imaginait des indicateurs de force des liens affectifs (comme la réaction face aux décès)... autant de démarches typiques de l'analyse de réseaux égocentrés ${ }^{14}$. Mais, sur de tels terrains, le problème de la comparabilité des sources paraît souvent insoluble - sauf à utiliser différentes périodes d'un même journal, comme le font les utilisateurs du logiciel Arcane, dédié aux "ego-documents ${ }^{15}$. L'apport des analyses formelles réside surtout dans un dialogue heuristique, qui met au jour des problèmes de définitions plus faciles à passer sous silence si l'on s'en tient à des études seulement qualitatives ${ }^{16}$.

Un autre type de sources de l'histoire sociale est souvent envisagé quand on parle d'analyse de réseaux: les actes d'état civil ou notariés. Ils permettent

13. Pour un approfondissement des thèmes de cette partie et une mise en rapport avec les approches moins formalisées de la notion de réseau, voir Claire LEMERCIER, "Analyse de réseaux et histoire de la famille: une rencontre encore à venir?», Annales de démographie historique, à paraître.

14. Alan MacFarlane, The Family Life of Ralph Fosselin, a Seventeenth-Century Clergyman: an Essay in Historical Anthropology, Cambridge, Cambridge University Press, 1970.

15. P.-Y. Beaurepaire, D. Taurisson (éd.), Les ego-documents..., op. cit. Voir notamment les articles utilisant efficacement des formalisations simples de Françoise DEROY-PINEAU et Paul BERNARD, "Projet mystique et stratégie réticulaire de mobilisation des ressources: Marie Guyart, mystique et stratège", p. 35-70; Stéphane HaFFEMAYER, "La correspondance de Nicolas Prunier de Saint-André, ambassadeur à Venise (1668-1672): limites et réalités du réseau d'influence d'un président au parlement de Grenoble», p. 71-96; Antony McKennA, Annie Leroux, "Les réseaux de correspondance de Pierre Bayle: réalité instable et représentation électronique", p. 399-420.

16. Cf. Karen V. HANSEN, Cameron L. MACDONALD, "Surveying the dead informant: quantitative analysis and historical interpretation", Qualitative Sociology, 18, 1995, p. 227-236, à propos de l'étude de visites mentionnées dans des journaux intimes d'ouvrier(e)s de Nouvelle-Angleterre au $\mathrm{XIX}^{\mathrm{e}}$ siècle. 
de constituer des réseaux égocentrés incluant des liens familiaux, voire non familiaux (par le biais des témoins). La lourdeur du dépouillement rend plus difficiles les études structurales. Mais surtout, une bonne compréhension de la construction de la source et de son degré d'homogénéité est essentielle. L'absence de mention d'une personne signifie-t-elle une absence de lien, ou plutôt une absence momentanée des lieux de l'acte ? Recourt-on à des témoins décrits comme "amis» en l'absence de parents (décédés ou éloignés géographiquement) ou parce que les amis sont plus proches affectivement? De telles questions interdisent de détacher l'analyse quantitative d'une connaissance fine de la source et du terrain ${ }^{17}$. Lorsqu'il est possible de mêler ces ressources, l'analyse en termes de réseaux s'avère toutefois féconde: on peut lui emprunter des concepts, comme Claire Dolan évoquant l'intermédiarité de certains acteurs au sein du milieu notarial d'Aix-en-Provence au XVI ${ }^{\mathrm{e}}$ siècle $^{18}$, ou des idées d'indicateurs, comme Paul-André Rosental mesurant le degré d'«autocentrage» de lignées françaises du XIX ${ }^{\mathrm{e}}$ siècle. Définissant comme autocentrées celles qui recourent souvent aux mêmes témoins et/ou pratiquent l'endogamie, il lie cette caractéristique "de réseau " à leurs comportements migratoires ${ }^{19}$.

Il est aussi possible d'utiliser l'analyse de réseaux pour réfléchir, à un niveau plus "méso" que "micro", à la sociabilité prise dans son sens premier, c'est-à-dire à l'intensité et à la structure des relations sociales à l'échelle de petites communautés comparées entre elles. C'est la stratégie que Sandro Lombardini utilise pour montrer la diversité des structures d'alliance en Italie au $\mathrm{XVII}^{\mathrm{e}}$ siècle et lutter ainsi contre des schémas culturalistes trop simples (il parle de "structures familiales floues» et de "communautés absentes»), tout en s'interrogeant sur les origines de la conflictualité locale ${ }^{20}$. Karen Barkey, plus intéressée par les révoltes paysannes (dans l'Empire ottoman à la même période), les lie à la structure des relations, familiales, amicales ou économiques, à l'intérieur et entre des villages, observée à partir d'archives judiciaires $^{21}$. Une équipe de l'université de Münster travaille sur les marchés fonciers en Westphalie au XIX ${ }^{\mathrm{e}}$ siècle en comparant, entre villages et au cours

17. Voir des réflexions souvent pessimistes dans Vincent GouRdon, Scarlett BEAUVALET, FrançoisJoseph RugGiU (dir.), Liens sociaux et actes notariés dans le monde urbain en France et en Europe, Paris, Presses de l'Université de Paris-Sorbonne, 2004 - et, en contrepoint, des résultats formalisés obtenus à partir de ce type de sources dans le numéro "Réseaux" à paraître dans Annales de démographie historique.

18. Claire Dolan, Le notaire, la famille et la ville. Aix-en-Provence à la fin du XvI siècle, Toulouse, Presses de l’Université du Mirail, 1998.

19. Paul-André Rosental, Les sentiers invisibles. Espace, famille et migrations dans la France du $X I X^{e}$ siècle, Paris, Éditions de l'EHESS, 1999.

20. Sandro Lombardini, «Family, kin, and the quest for community: a study of three social networks in early-modern Italy", History of the Family, 1/3, août 1996, p. 227-257, citation p. 230 (fondé seulement sur les réseaux d'alliances, utilisant toutes les méthodes d'analyse structurale).

21. Karen BARKEY, "The uses of court records in the reconstruction of village networks: a comparative perspective", International fournal of Comparative Sociology, 32/1-2, 1991, p. 196-216 (présentation des données) et Karen BARKeY, Ronan vAN Rossem, "Networks of contention: villages and regional structures in the seventeenth-century Ottoman Empire", American fournal of Sociology, 102/5, mars 1997, p. 1345-1382 (résultats). 
du siècle, les liens de parenté, alliance, crédit et achat de terres - définis de façon très rigoureuse ${ }^{22}$. Dans tous les cas, les comparaisons (entre liens, lieux, périodes) enrichissent l'analyse. Intégrés dans des problématiques différentes - sur les systèmes d'alliances, le rapport à l'État ou les relations entre réseaux personnels et marchés -, ces trois recherches démontrent que l'on peut, à partir des sources classiques de l'histoire sociale (état civil, actes notariés, cadastre, archives judiciaires), réaliser des analyses de réseaux très fécondes, permettant de caractériser une structure sociale à l'échelle moyenne de la petite communauté.

\section{Au-delà des généalogies? Repenser les représentations des lignées}

Les historiens peuvent donc, en complément de travaux plus qualitatifs ou plus classiquement quantitatifs, s'emparer d'outils issus de l'analyse de réseaux. Celle-ci peut aussi avoir une fonction de "dénaturalisation" des représentations. L'apparente évidence des arbres généalogiques est en effet trompeuse, on s'en aperçoit si on les reformule en termes de réseaux: doit-on considérer les relations au nième degré comme une composition de relations au premier degré? Comment prendre en compte la force particulière de certains liens selon la société étudiée (ainsi, l'oncle maternel et l'oncle paternel peuvent très bien ne pas tenir des fonctions symétriques) ? À ces questions familières des anthropologues, l'un d'eux, Douglas R. White, a proposé des réponses originales. Par exemple, il considère les unions reproductives comme des points et les individus comme des liens entre ces points ${ }^{23}$. Ce renversement, qui évite certains présupposés culturels, permet aussi de visualiser facilement les «renchaînements d'alliances" entre lignées et de donner plusieurs définitions différentes et rigoureuses de la notion d' "endogamie»"

D'autres "réseauistes" ont proposé des pistes pour une histoire culturelle de la famille. Peter S. Bearman, travaillant sur des élites locales anglaises des $\mathrm{XVI}^{\mathrm{e}}-\mathrm{XVII}^{\mathrm{e}}$ siècles, a ainsi choisi de prendre au sérieux le discours des généalogies de l'époque, ce qui l'a amené à considérer les liens entre lignées comme asymétriques $^{25}$. En effet, dans ses sources, les familles les moins considérées

22. Georg FERTIG, «Zwischen Xenophobie und Freundschaftspreis: Landmarkt und familiäre Beziehungen in Westfalen, 1830-1866", Fahrbuch für Wirtschaftsgeschichte, Heft 1, 2005 (premier article émanant d'un projet collectif, "Soziale Netzwerke in der ländlichen Gesellschat", portant notamment sur le rôle de la "confiance" et du "capital social» en histoire économique).

23. Douglas R. White, Paul Jorion, "Kinship networks and discrete structure theory: applications and implications", Social Networks, 18/3, août 1996, p. 267-314, avec quelques exemples historiques.

24. Sur l'endogamie: Lilyan BRUDNER, Douglas WHITE, "Class, property and structural endogamy: visualizing networked histories", Theory and Society, 26/2-3, avr.-juin 1997, p. 161-208, retrace l'histoire matrimoniale et patrimoniale de 2000 couples ayant vécu dans le village autrichien de Feistritz de 1850 à 1960 .

25. Peter S. Bearman, Relations into Rhetorics. Local Elite Structure in Norfolk, England, 15401640, New Brunswick, Rutgers University Press, 1993 - ouvrage par ailleurs très formaliste, représentant d'une vision du réseau comme paradigme qui peut ne pas convaincre. 
donnaient les plus grands détails sur leurs parentés, même lointaines, avec d'autres d'un statut plus élevé, tandis que ces dernières s'en tenaient à des arbres patrilinéaires minimaux. En prendre conscience permet d'étudier des «affirmations de parenté» (de moins en moins asymétriques avec le temps). Ainsi, en traitant les liens familiaux comme tous les autres, en prenant de la distance par rapport à l'objectivisme coutumier en la matière, l'analyse de réseaux permet de réfléchir autrement sur la place des lignages ou des alliances dans les sociétés du passé.

\section{Réseaux sociaux et modernisation}

Même si l'on souhaite utiliser l'analyse de réseaux comme un simple outil, se pose souvent, en particulier en histoire sociale, la question de l'évolution réelle des structures relationnelles, à un niveau macro; pour éviter une vision caricaturale de l'"archaïsme" et de la "modernité", il faut sans doute, avant toute analyse en termes de réseaux, préciser des hypothèses sur ce point. L'idée classique est en effet que la "modernisation» s'accompagnerait de la disparition des "communautés" et de l'essor de rapports sociaux plus "privés", moins contraints par des appartenances statutaires (sociales, ethniques ou autres). Une tradition américaine de Community Studies s'est ainsi attachée à exploiter, de façon en général non formalisée, toutes les sources nominatives officielles et/ou privées pour comprendre par exemple si les relations restaient limitées ou non à un rayon géographique étroit ${ }^{26}$. Certains chercheurs souhaitent aller vers de véritables études comparées avec la sociologie des réseaux contemporains, affirmant que l'analyse de réseaux sociaux traite de "questions atemporelles» (arguably timeless matters) telles que la densité, la multiplexité ou la localisation géographique des réseaux personnels. Mais les problèmes de sources réduisent la portée de leurs travaux, les ramenant à des études plus classiques, comme celle du nombre de parents présents sur place ${ }^{27}$. Il paraît tout aussi difficile de postuler, à l'inverse, une totale incommensurabilité entre les liens sociaux d'aujourd'hui et ceux du passé. Ainsi, J. M. Imizcoz Beunza reprend les positions de Louis Dumont sur les sociétés holistes opposées aux «sociabilités démocratiques", considérant en particulier que les liens de l'Ancien Régime n'impliquent pas d'adhésion libre et révocable de la part des individus ${ }^{28}$.

26. On peut distinguer la belle étude de Darrett B. Rutman, Anita H. Rutman, A Place in Time: Middlesex County, Virginia, 1650-1750, New York, Norton, 1984, fondée sur une véritable "prosopographie de masse" faisant feu de tout bois, intégrant des concepts issus de l'analyse de réseaux et très nuancée en termes de conclusions sur la modernisation.

27. Charles Wetherell, Andrejs Plakans, Barry Wellman, «Social networks, kinship, and community in Eastern Europe", Fournal of Interdisciplinary History, 24/4, printemps 1994, p. 639-663 (citation p. 650); Barry WeLlman, Charles WeTHERELL, "Social network analysis of historical communities: some questions from the present for the past", History of the Family, 1/1, janv. 1996, p. 97-121; Charles WETHERELL, "Historical social network analysis", International Review of Social History, 43, supplement 6, 1998, p. 125-144.

28. José Maria Imizcoz Beunza, "Communauté, réseau social, élites. L'armature sociale de l'Ancien Régime", in J. L. Castellano, J.-P. Dedieu (dir.), Réseaux, familles..., op. cit., p. 31-66. 
Il semble pourtant que réfléchir à partir des concepts de l'analyse de réseaux, mais en adaptant la définition des liens et le choix des sources à la société étudiée, peut apporter quelque chose de nouveau. En matière de stratification sociale, de véritables travaux d'histoire ont ainsi pu utiliser soit des graphes, soit des indicateurs plus élaborés pour statuter sur la perméabilité de groupes sociaux (chevaliers et sénateurs de Rome) ou ethniques (Indiens, Espagnols et métis du XVII ${ }^{\mathrm{e}}$ siècle $)^{29}$. Ce succès se fonde sur l'usage précautionneux de sources relativement riches - correspondance de Cicéron ou testaments.

D'autre part, en matière d'histoire des élites, à la fois sociale et politique, utiliser des méthodes formalisées peut aider à penser un "réseau» qui ne soit pas seulement un groupe fermé et densément relié, une communauté close et homogène, mais d'envisager quelque chose d'en partie ouvert (à un degré mesurable), de hiérarchisé, de spécialisé. À partir de correspondances, Thierry Rentet a ainsi reconstitué les relations complexes entre les officiers d'Anne de Montmorency: une très simple analyse de réseaux complète son travail qualitatif et quantitatif détaillé; surtout, il utilise les concepts de l'analyse structurale de façon critique, pour refuser une vision trop simple des factions ou clientèles ${ }^{30}$. La formalisation lui permet d'insister sur le recoupement entre différents types de liens, formels ou informels, ou encore d'identifier des "intermédiaires clés" au sein du réseau. Il apparaît ainsi possible d'utiliser l'analyse de réseaux pour nuancer une vision trop rigide des sociétés passées, sans pour autant tomber dans l'anachronisme.

\section{GÉOGRAPHIE HISTORIQUE, HISTOIRE DES SAVOIRS, HISTOIRE DES ENTREPRISES: DES APPLICATIONS " UTILITAIRES » E L'ANALYSE DE RÉSEAUX}

Les sources classiques de la prosopographie peuvent donc être mobilisées pour reconstituer des liens, en général dans la perspective de réseaux égocentrés et comme simple complément de démarches plus classiques. Dans certaines sous-disciplines historiques, l'analyse de réseaux peut être appliquée plus systématiquement à des sources déjà bien structurées en termes de relations. L'objet de l'étude n'est alors plus le lien social: l'analyse de réseaux apparaît comme un simple outil descriptif, utile pour résumer la structure d'ensembles complexes de liens. Si ces démarches ne sont pas spécifiques à l'histoire, les appliquer à des sources disponibles sur de longues périodes peut produire des résultats intéressants à la fois pour l'histoire et pour des disciplines liées: géographie, histoire des sciences ou du droit, économie, gestion.

29. Michael C. AlexAnder, James A. Danowski, «Analysis of ancient networks: personal communications and the study of social structure in a past society", Social Networks, 12/4, déc. 1990, p. 313-335; Jacques POLONI-SimARD, "Liens personnels et milieux sociaux dans une société coloniale de l'Audience de Quito, 1620-1680", in J. L. Castellano, J.-P. Dedieu (dir.), Réseaux, familles..., op. cit., p. 191-230 et La mosä̈que indienne. Mobilité, stratification sociale et métissage dans le corregimiento de Cuenca (Équateur) du XVI ${ }^{e}$ au XVIII siècle, Paris, Éditions de l'EHESS, 2000.

30. Thierry RENTET, «Network mapping: ties of fidelity and dependency among the major domestic officers of Anne de Montmorency", French History, 17/2, juin 2003, p. 109-126. 


\section{Système-monde, systèmes locaux, échanges de biens et de populations}

Plutôt qu'à des individus, il est possible d'appliquer l'analyse de réseaux à des unités spatiales plus ou moins larges - tout en gardant en tête le fait que ce ne sont pas des lieux qui réalisent les échanges de marchandises ou de populations. L'analyse de réseaux est particulièrement adaptée aux raisonnements portant sur des flux: elle permet de mettre en évidence des goulets d'étranglement, des intermédiaires privilégiés, des périphéries délaissées ou des centres secondaires autonomes.

Ainsi, c'est très tôt qu'elle a été employée pour tester les théories du «système-monde» et de l'échange inégal d'Immanuel Wallerstein (décrivant une division en trois cercles concentriques, accompagnée d'une forte différenciation des échanges commerciaux selon les produits). L'analyse structurale de l'équivalence produit en effet une classification en "positions" permettant un test direct de ce type d'hypothèse. Ainsi, D. Smith et D. White ont pu discuter l'évolution de la structure globale des échanges, mais aussi, en changeant d'échelle, de la position de tel ou tel pays en son $\operatorname{sein}^{31}$. Des travaux en cours de M. Flandreau et C. Jobst mettent en œuvre la même stratégie pour clarifier les notions intuitives de "centre" et "périphérie" à propos des monnaies de la fin du XIX ${ }^{\mathrm{e}}$ siècle. Ils mobilisent toutes les techniques de l'analyse de réseaux à partir d'une source originale: les mentions mutuelles de places de change dans les journaux financiers $^{32}$. La même invention en matière de sources se retrouve pour les études de flux économiques portant sur des périodes pré-statistiques: l'analyse formelle des réseaux de transports peut ainsi aider à comprendre les dynamiques de localisation sans céder aux déterminismes physiques ${ }^{33}$.

Du côté des migrations, question formellement comparable, il est également possible d'imaginer des stratégies réplicables pour rendre compte de façon relationnelle de la "micro-mobilité», en particulier ${ }^{34}$. L'analyse en termes de réseaux (plus précisément, d'équivalence structurale) permet en effet de tester l'hypothèse d'une mobilité aléatoire, fondée seulement sur les dimensions de villes et les distances, et celles, concurrentes, de "pays» fermés, de lieux liés par des migrations en chaîne préférentielles ou d'un simple exode

31. David A. Smith, Douglas R. White, «Structure and dynamics of the global economy: network analysis of international trade 1965-1980", Social Forces, 70/4, juin 1992, p. 857-893 - article très riche en termes de méthodes.

32. Ces travaux ont été présentés dans diverses conférences, dernièrement à Barcelone (janvier 2005), au $10^{\mathrm{e}}$ symposium d'histoire économique sur "L'analyse de réseaux en histoire économique", sous le titre "The ties that divide. A network analysis of the international monetary system, 1890-1910".

33. Forrest R. PITTs, "The medieval river trade network of Russia revisited", Social Networks, vol. $1, \mathrm{n}^{\circ} 3,1978-1979$, p. 285-292; David JENkINS, "A network analysis of Inka roads, administrative centers, and storage facilities", Ethnohistory, 48/4, automne 2001, p. 655-687.

34. C. Lemercier, P.-A. Rosental, "Pays” ruraux et découpage de l'espace: les réseaux migratoires dans la région lilloise au milieu du XIX ${ }^{\mathrm{e}}$ siècle", Population, 55/4-5, juill.-oct. 2000, p. 691-726. 
rural. Elle propose une réponse nuancée, mettant au jour aussi bien une frontière linguistique dans la région étudiée que la situation particulière de chaque ville. Cette analyse est fondée sur un changement d'échelle dans le traitement des sources, l'agrégation par commune d'actes de mariage (fournissant lieux de naissance et de résidence) permettant de constituer des liens entre lieux, sans interdire un retour aux trajectoires individuelles "exceptionnelles» susceptible d'enrichir les conclusions.

Assez facilement réplicables - sans qu'il faille pour autant minimiser les problèmes liés à l'interprétation des sources -, ces études proposent donc des outils complémentaires pour répondre à des questions de recherche liées à des systèmes d'échanges ou de circulations.

\section{"Scientométrie» et histoire des savoirs scientifiques ou juridiques}

De même, les études de citations peuvent être considérées comme un outil complémentaire de test d'hypothèses. Ce domaine a bénéficié, pour son financement, de son utilisation croissante pour l'évaluation de la recherche (les célèbres «indices d'impact» ou «index de citations») ${ }^{35}$. De ce fait, il a mauvaise presse auprès des chercheurs, qui en perçoivent immédiatement les limites: en particulier, une citation n'est pas forcément positive et les phénomènes de centralité ont tendance à s'auto-entretenir. La méthode est en outre extrêmement dépendante du choix du corpus ${ }^{36}$. Ces critiques invitent plutôt à proposer des usages plus nuancés de la technique, potentiellement utile dans tous les domaines de l'histoire des savoirs. Ainsi, avec une méthode originale, D. White et H. Gilman McCann ont pu proposer une définition argumentée de la «révolution chimique» du XVIII ${ }^{\mathrm{e}}$ siècle comme changement de paradigme ${ }^{37}$. Analysant 3000 citations d'une centaine d'auteurs, ils regardent non pas "qui cite qui», mais "qui est cité (plus ou moins systématiquement) avec qui", pour savoir s'il est possible de hiérarchiser les chimistes et de définir des écoles de pensée. Apparaît ainsi une reconfiguration dans le temps du réseau de cocitations, avec une période intermédiaire assez confuse et la construction d'un nouveau consensus.

Permettant de définir des acteurs centraux ou de discuter des limites entre disciplines, la scientométrie peut s'appliquer à d'autres domaines où la citation joue un grand rôle, en particulier à l'étude de textes juridiques. Sur de telles données, relativement simples à définir, toute la gamme des techniques de

35. Pour un tour d'horizon: Michel Callon, Jean-Pierre Courtial, Hervé Penant, La scientométrie, Paris, PUF, 1995.

36. Ainsi, malgré son raffinement technique, l'article de Norman P. Hummon, Patrick Doreian, "Connectivity in a citation network: the development of DNA theory", Social Networks, 11/1, mars 1989 , p. 39-63 souffre de la délimitation a priori du corpus à partir de travaux de vulgarisation, amenant à des conclusions assez tautologiques.

37. D. R. White, H. Gilman McCanN, "Cites and fights: material entailment analysis of the Eigtheenth-Century chemical revolution", in Barry WELLMAN and Steven BERKowITZ, Social Structures : a Network Approach, Cambridge, Cambridge University Press, 1988, p. 380-399. 
l'analyse de réseaux peut être utile: mesures de la centralité de telle ou telle cour, recherche de "cliques", régionales ou non, de cours se citant entre elles et ignorant relativement les autres, ou encore de centres et de périphéries... Mener des études comparables à celles qui existent sur les cours suprêmes américaines $^{38}$ à propos de pays au système judiciaire a priori plus centralisé et hiérarchisé serait certainement très intéressant.

\section{Interlocking directorates et histoire des entreprises}

L'étude des liens entre conseils d'administration (créés par l'existence de membres communs) a, elle, depuis les années 1980, connu une véritable fortune qui rend un survey exhaustif impossible, même si l'on se limite aux données historiques; celles-ci sont parfois utilisées de façon seulement instrumentale par des spécialistes de gestion peu intéressés par l'histoire sociale des élites ou l'évolution des contextes institutionnels. Il reste toutefois bien des travaux proprement historiques dans ce domaine de recherche, qui a en réalité une double origine. Très tôt, les interlocks ont été comptés - et pourchassés par les commissions antitrust américaines ${ }^{39}$. Dans une perspective en partie comparable, des chercheurs ont décrit et dénoncé, dans les années 1970, le caractère fermé des élites américaines et la superposition de leurs réseaux de différents types. Parallèlement, les interlocks ont intéressé les gestionnaires qui discutaient du pouvoir dans la firme (managérial ou actionnarial?) et du rôle des banques dans l'économie ${ }^{40}$. De ces préoccupations variées ont émergé des recherches inégales et parfois trop formalistes, ce que facilitait le caractère standardisé des sources. Certaines négligent des points importants, comme les chronologies de prises de fonctions (un cumul est rarement instantané), le poids des compétences ou des liens privés des individus concernés (loin de représenter un simple lien interchangeable entre firmes) ou la réalité du travail des conseils et de l'implication de chacun de leurs membres - éléments que l'outil «réseaux", en lui-même, n'interdit pourtant pas de prendre en compte ${ }^{41}$.

D'autres chercheurs ont considéré à la fois les parcours individuels, les normes juridiques et les stratégies économiques, et cette perspective parait se

38. Cf. en particulier Peter HARRIS, «Structural change in the communication of precedent among State Supreme Courts, 1870-1970", Social Networks, 4/3, sept. 1982, p. 201-212, pour son caractère historique et, pour une présentation de la stratégie de recherche, Gregoy A. CALDEIRA, "Legal precedent: structures of communication between State Supreme Courts", Social Networks, 10/1, mars 1988, p. 29-55.

39. Voir la description et la reformulation des tableaux et graphiques d'un rapport de $1916 \mathrm{du}$ Département de l'Agriculture dans William J. HAUSMAN, «Webs of influence and control: personal and financial networks in the formative years of the US electric power industry", Annales historiques de l'électricité, $\mathrm{n}^{\circ} 2$, juin 2004, p. 53-67.

40. Pour un bilan général, voir Mark S. MizRuchI, «Berle and means revisited: the governance and power of large corporations", Theory and Society, 33/5, oct. 2004, p. 579-617.

41. Mark S. MizRUCHI, "What do interlocks do? An analysis, critique, and assessment of research on interlocking directorates", Annual Review of Sociology, 22, 1996, p. 271-298. 
répandre dans différents pays, si l'on en juge par de récents colloques ${ }^{42}$. Pourtant bien plus ancien, un livre de Mark S. Mizruchi fait encore référence: véritable étude de longue durée, très clair en termes de méthodes, il change en permanence d'échelle, des trends sur tout le siècle à l'impact immédiat de la loi Clayton de 1914 ou aux stratégies de la banque Morgan, et se confronte aux perceptions des contemporains ${ }^{43}$. Du côté français, une étude récente marie efficacement histoires sociale et économique en étudiant les implications collectives variées de patrons marseillais, en complément de très riches sources plus «qualitatives"; l'objectif est ici de cerner les origines de la "concurrence coopérative" qui se maintient au sein de ce groupe ${ }^{44}$. La forte densité globale des liens n'y annihile pas frontières et hiérarchies internes. À l'échelle individuelle, l'auteur évoque la multiplication des implications, qui expliquerait l'efficacité différentielle des propriétés sociales selon les personnes - par exemple le fait que certains représentants de familles récemment implantées atteignent un statut plus élevé que d'autres, une bonne stratégie relationnelle compensant le manque d'autres attributs. L'étude des positions multiples se place dans la lignée d'analyses classiques des élites et du capital social ${ }^{45}$; mais l'analyse de réseaux permet plus de finesse que le simple comptage des positions occupées par chaque individu.

Avec les conseils d'administration et leurs membres "cumulards", on touche à un type de sources plus facile à exploiter en termes d'analyse de réseaux que celles qui sont censées indiquer directement un lien interpersonnel (correspondances, journaux intimes...): il s'agit là plutôt d'un engagement conjoint. En effet, on peut tirer de simples listes de membres de diverses institutions, entreprises ou associations des liens (parfois appelés «liens indirects») soit entre personnes, soit entre institutions. Pour éviter toute surinterprétation, il faut bien sûr réfléchir sur le sens de la co-présence (comme de la co-citation en histoire des savoirs), qui n'implique pas forcément d'interaction réelle, voire pas d'inter-connaissance. À cette condition, de telles analyses, également applicables aux mouvements sociaux ou politiques, s'avèrent très fécondes.

42. En particulier celui, précité, de Barcelone, où ont été présentés les travaux de thèse réalisés à Stanford par Aldo Musacchio et Armando Razo sur le Mexique et le Brésil autour de 1900 et non encore publiés, travaux qui prennent en compte à la fois les dimensions économique, institutionnelle et sociale (avec le rôle des élites politiques) de la question. Une étude très riche est également en cours en Suisse (Thomas David, Martin Lüpold, André Mach et Gerhard Schnyder). Parmi les travaux plus classiques, un des plus intéressants en termes d'histoire et des plus clairs en termes de méthode est celui de William G. Roy, "The unfolding of the interlocking directorate structure of the United States", American Sociological Review, 48/2, avr. 1983, p. 248-257 (sur la période 1886-1905).

43. Mark S. Mizruchi, The American Corporate Network: 1904-1974, Beverly Hills, Sage, 1982.

44. Pierre-Paul ZaLIO, «Un monde patronal régional dans les années 1930. Une perspective configurationnelle", Cahiers lillois d'économie et de sociologie, $\mathrm{n}^{\circ} 41$ : «Repenser le marché», Bernard CONVERT (dir.), Paris, L'Harmattan, 2003, p. 137-176. Quelques dizaines d'associations, institutions ou conseils d'administration, regroupant environ 500 individus, sont pris en compte, les conseils d'administration étant distingués pour une partie de l'analyse.

45. Comme celles de Luc Boltanski, «L'espace positionnel. Multiplicité des positions institutionnelles et habitus de classe", Revue française de sociologie, 14/1, janv.-mars 1973, p. 3-26, et Pierre Bourdieu, La noblesse d'État, Paris, Minuit, 1989. 


\section{INDIVIDUS ET ORGANISATIONS EN HISTOIRE POLITIQUE}

Les applications de l'analyse de réseaux à des données historiques se sont le plus souvent faites en ordre dispersé; une véritable école, avec des résultats plus cumulatifs, a toutefois émergé dans le domaine de l'étude des "mouvements sociaux", qui regroupe des sociologues et des politistes souvent influencés par Charles Tilly et accordant une certaine attention à l'histoire ${ }^{46}$. Dans ce champ de recherche, la notion de réseau intervient à deux niveaux. D'une part, à l'échelle individuelle, l'inscription relationnelle est envisagée comme l'un des déterminants de l'engagement dans des mouvements sociaux ou politiques, de la "mobilisation». Cette tradition d'analyse qui a quelques décennies $^{47}$ se renouvelle aujourd'hui en lien avec des courants plus centrés sur les cultures, les langages, les "significations». D'autre part, les réseaux d'organisations sont pris en compte: échanges, actions communes ou partages de membres entre des groupes eux-mêmes plus ou moins formellement organisés. La notion de réseau permet ainsi de mettre en question le concept de "mouvement social», flou de l'aveu même de ses utilisateurs ${ }^{48}$, en spécifiant le caractère plus ou moins formel et plus ou moins fermé des organisations et groupes d'organisations.

\section{Expliquer des engagements autrement que par des attributs individuels}

La première génération des analyses de la mobilisation en termes de réseaux s'est surtout attachée aux déterminations de choix individuels (s'engager ou non, dans un camp ou dans l'autre, poursuivre ou non cet engagement), mettant en évidence le fait que ces choix sont en partie liés à une position définie en termes de réseaux sociaux, et non pas seulement à des attributs individuels plus classiques (niveau social, âge, sexe...). Cette lutte contre un usage naïf des "catégories" a eu son mérite et ses dérives (le réseau étant supposé annihiler toute notion de classe, par exemple); aujourd'hui, les frontières entre "réseau" et "attributs" sont reconnues comme plus floues, la notion d'«identité» permettant de les relier.

Les textes pionniers ont adopté une stratégie démonstrative commune, reprenant les explications "classiques» de tel ou tel choix, les testant (souvent par une régression) sans trop de succès, puis tirant d'une analyse de réseaux un nouvel attribut, reflétant une position relationnelle (centralité, position dans telle ou telle clique...), et qui s'avérait plus «explicatif» de l'engagement final.

46. En témoignent des livres collectifs comme celui de Mario Diani, Doug McAdam (eds), Social Movements and Networks. Relational Approaches to Collective Action, Oxford-New York, Oxford University Press, 2003, qui fédère des approches variées et fournit des synthèses, en particulier en introduction et au chapitre 13 .

47. Avec pour référence notamment David A. SNow, Louis A. ZurCHER, Sheldon EkLAND-Olson, "Social networks and social movements : a microstructural approach to differential recruitment", American Sociological Review, 45/5, oct. 1980, p. 787-801.

48. Erik Neveu, Sociologie des mouvements sociaux [1996], Paris, La Découverte, 2002. 
Mené de façon plus ou moins subtile ${ }^{49}$, ce type de raisonnement a été également à la base d'un article justement célèbre, qui intègre une vision plus historique des événements. Il ne relève pas de l'étude des "mouvements sociaux" proprement dits, puisqu'il porte sur l'arrivée au pouvoir des Médicis à Florence, au XV $\mathrm{XV}^{\mathrm{e}}$ siècle $^{50}$. Il s'agit de comprendre d'une part la répartition des élites entre soutien aux Médicis et à leurs adversaires, d'autre part le succès des premiers, dont l'hétérogénéité du point de vue social et économique est bien connue. Des données sur les alliances matrimoniales et les liens économiques entre familles, soumises à une analyse d'équivalence structurale, montrent l'importance du rôle d'intermédiaires exclusifs des Médicis et du fait qu'ils cloisonnent leurs réseaux. Leurs adversaires, eux - avec lesquels ils contractent des mariages -, constituent une clique densément reliée, par des liens à la fois familiaux et économiques, et peu hiérarchisée, ce qui peut contribuer à expliquer leurs rivalités internes. L'article évoque aussi la construction de cette configuration particulière et s'interroge sur le degré auquel elle a été consciente de la part des Médicis. Le problème du lien entre réseaux et stratégies, du fait que les structures émergent ou non d'un ensemble de comportements individuels tendus vers d'autres buts, est donc pris au sérieux. L'évolution des mariages sur un siècle montre que la clique a émergé en réaction à la révolte des Ciompi, peut-être en lien avec une nouvelle conscience aristocratique (remplaçant des unions localisées par quartier). Les Médicis n'auraient délibérément exploité ce phénomène que bien plus tard. «De façon presque hégélienne, les oligarques créèrent des réseaux menant à leur propre destruction", concluent les auteurs ${ }^{51}$. Si leurs résultats sont sujets à débat, la méthode proposée, avec son attention à diverses échelles (des choix individuels aux révoltes ou guerres), ne peut qu'intéresser les historiens.

La même subtilité se retrouve dans l'étude menée par le politiste John P. Willerton sur un terrain bien différent: il décrit l'évolution des milieux dirigeants de l'URSS brejnévienne ${ }^{52}$. L'étude porte sur des liens de "patronage», définis de façon objectiviste (en fonction des carrières parallèles des patrons et clients) mais interprétés grâce à une connaissance fine du terrain et des personnages. Comme celui de John F. Padgett et Christopher K. Ansell, l'article ne se borne pas à identifier un «réseau Brejnev» qui serait une faction à

49. Pour un raisonnement poussé à l'absurde, du fait de sources très limitées, voir R. V. Gould, "Patron-client ties, state centralization, and the whiskey rebellion", American fournal of Sociology, 102/2, sept. 1996, p. 400-429; pour une étude efficace fondée sur des sources très riches, mais sans analyse de réseaux au sens strict, voir Peter S. BEARMAn, "Desertion as localism: army unit solidarity and group norms in the US Civil War", Social Forces, 70/2, déc. 1991, p. 321-342.

50. John F. PADGETT, Christopher K. ANSELL, "Robust action and the rise of the Medici, 1400-1434", American fournal of Sociology, 98/6, mai 1993, p. 1259-1319.

51. Ibid., p. 1287. Cette étude des alliances a été affinée dans J.F. PADGETT, "Marriage and elite structure in Renaissance Florence, 1282-1500", Paper delivered to the Social Science History Association, 1994 (téléchargeable sur le web).

52. John P. WILleRTON, "Patronage networks and coalition building in the Brezhnev era", Soviet Studies, 39/2, avr. 1987, p. 175-204; J. Willerton, Patronage and Politics in the USSR, Cambridge, Cambridge University Press, 1992. 
part et qui connaîtrait plus de succès que les autres. Les différences entre sous-groupes du réseau, tant en termes de structure interne (plus ou moins pyramidale) que de répartition dans différentes régions ou fonctions sont analysées; leur efficacité ne se comprend qu'en lien avec une étude plus institutionnelle des leviers de pouvoir. Le système d'alliances entre factions, la capacité à construire un consensus sont également pris en compte. Il s'agit de rechercher les «positions organisationnelles permettant de structurer l'agenda politique $»^{53}$.

Ainsi, l'étude en termes de réseaux sociaux peut permettre de mieux comprendre des comportements impliquant un choix fait à un moment précis - choix d'engagement ou encore d'adoption d'une innovation ${ }^{54}$ - sans pour autant imposer un "déterminisme du réseau", du moins si l'on prend en compte sa nature mouvante et les autres éléments de contexte qui orientent les choix.

\section{Réseaux, identités et discours}

Malgré leurs nuances, les analyses précédentes conservent à la structure des liens, voire aux carrières ou aux institutions, un poids assez déterminant. En parallèle, la floraison d'analyses en termes de réseaux a créé une certaine insatisfaction face à un caractère parfois trop formaliste, déterministe, enlevant tout poids aux appartenances à des groupes ou encore à la question de la vision des acteurs et du caractère conscient des stratégies ${ }^{55}$. En a découlé une meilleure prise en compte, dans des travaux postérieurs, de l'articulation entre réseaux et identités, dorénavant décrite par certains en termes de construction mutuelle. Parallèlement, les discours eux-mêmes ont fait l'objet d'analyses en termes de réseaux, participant d'une histoire plus culturelle des mouvements sociaux.

Après une période où la "relation" a été brandie en opposition à l'«attribut" (vu comme une catégorie souvent artificielle et peu explicative), l'accent est plutôt mis aujourd'hui sur le flou des frontières entre les deux notions - permettant d'éviter l'assimilation de l'attribut à la classe et du réseau au refus du concept de classe. En effet, si l'attribut peut être compris comme l'appartenance à un groupe décrit comme homogène et la relation comme quelque chose de plus électif, spécifique et choisi, dans les faits, les barrières sont bien plus floues. Par exemple, si définir un individu comme "ouvrier» ne nous dit à peu près rien sur son insertion relationnelle, le définir comme "polytechnicien"

53. J. Willerton, "Patronage...», art. cit., p. 189

54. On pense notamment à l'étude pionnière d'Alberto Maria BANTI, Terra e denaro. Una borghesia padana dell'Ottocento, Venise, Marsilio Editori, 1989, chap. 5 et 6, qui étudie la diffusion d'un nouveau comportement économique (l'usage d'engrais) à partir de liens sociaux préexistants et de façon formalisée, mettant notamment en exergue le rôle des "intermédiaires".

55. M. Emirbayer, J. Goodwin, "Network analysis...", art. cit. Dans cette présentation d'ensemble, deux spécialistes de la sociologie "culturelle» des mouvements sociaux mettent en évidence les modèles causaux implicites dans plusieurs articles (plutôt historiques) utilisant l'analyse de réseaux. 
lui attribue déjà un ensemble potentiel de liens (activables dans certaines circonstances). C'est le passage du simple attribut à une identité consciente, au nom de laquelle on s'engage, qu'ont étudié en particulier Doug McAdam (travaillant sur les mobilisations pour les droits civiques dans les années 1960, surtout à partir d'entretiens) et Roger V. Gould, notamment à propos de la Commune de Paris ${ }^{56}$. Au-delà des conclusions précises proposées sur leurs terrains, ils donnent une vision dynamique de l'articulation entre réseaux et identités, dans le cas particulier des mouvements sociaux. Ils insistent sur la notion de choix d'une identité de mobilisation, relativement prééminente, parmi un répertoire d'identités possibles et éventuellement concurrentes. La notion d'identité recouvrant notamment la désignation d'"amis» et d' "ennemis", les liens sociaux interviennent dans sa définition. L'engagement est vu comme un choix progressif de hiérarchisation entre identités, impliquant entre autres la réception d'informations sur la mobilisation, des arbitrages entre identités et réseaux concurrents, l'influence d'un encadrement par des structures collectives plus formalisées... En outre, bien évidemment, cet engagement reconfigure lui-même le réseau et tous deux sont influencés par les événements extérieurs.

John F. Padgett, dans la suite de ses recherches sur les Médicis, a également tenu compte des réserves exprimées à propos de son article précité. Certains soulignaient ainsi que, s'il était intéressant de montrer que les partisans des Médicis n'étaient pas réellement des "hommes nouveaux", il fallait pour autant comprendre pourquoi beaucoup d'entre eux se caractérisaient ainsi ou pourquoi leurs adversaires leur attribuaient ce qualificatif. Poursuivant la construction d'une base de données relationnelle énorme sur Florence entre 1340 et 1480 (elle comprend plusieurs dizaines de milliers de relations de types variés impliquant les élites urbaines), John F. Padgett intègre de plus en plus dans sa démonstration les aspects d'identité et de langage. Ainsi, la manière dont on en vient à se penser comme banquier ou comme popolani ou à désigner ses adversaires comme oligarques est envisagée en fonction des interactions entre groupes autant que de chocs extérieurs (guerres, révoltes...), les effets de ceux-ci suivant des lignes de faiblesse des réseaux sociaux de chaque groupe. La recherche porte aussi sur la notion d'«intérêts", l'accent étant mis sur la multiplicité des facettes individuelles: les

56. Voir M. Diani, D. McAdam (eds), Social Mouvements..., op. cit., en particulier les chap. 10 (R. V. GoulD, "Why do networks matter? rationalist and structuralist interpretations») et 12 (D. MCADAM, "Beyond structural analysis: toward a more dynamic understanding of social movements"); D. MCADAM, Ronnelle PAULSEN, "Specifying the relationship between social ties and activism", American Fournal of Sociology, 99/3, nov. 1993, p. 640-667; R. V. Gould, Insurgent Identities. Class. Community and Protest in Paris from 1848 to the Commune, Chicago, University of Chicago Press, 1995, notamment l'introduction. Pour une analyse de réseaux formalisée, voir R.V. GouLD, «Multiple networks and mobilization in the Paris Commune, 1871 ", American Sociological Review, 56/6, déc. 1991, p. 716-729, qui met en évidence l'influence des individus engagés dans la Garde nationale hors de leur quartier sur la diffusion de la mobilisation. 
acteurs jouent plusieurs jeux ${ }^{57}$, chacun avec leur objectif (le profit, le pouvoir, de nombreux enfants...), ce qui peut rendre leurs actions imprévisibles. Ces réflexions amènent à réexaminer des questions précises dans le cas de Florence, comme la diffusion de formes d'organisation des sociétés ou d'innovations comptables ${ }^{58}$.

Parallèlement à ces études mettant l'accent sur les rapports entre identités et liens sociaux, une autre stratégie de recherche a été poursuivie pour prendre en compte les langages et les cultures dans l'étude des mouvements sociaux. Il s'agit là de soumettre le discours lui-même à une analyse en termes de réseaux, pour reconstituer des relations entre groupes sociaux, organisations ou institutions, telles qu'elles sont exprimées et non «telles qu'elles existent en réalité». Les événements relatés dans les sources peuvent en effet être codés en termes d'actions exercées par un sujet vis-à-vis d'un objet (par exemple: «les habitants de Manchester interpellent la Chambre des communes»), puis traités comme des relations orientées (ici, une relation d'interpellation). On obtient ainsi un réseau de liens et de non-liens entre groupes pour chaque relation, telle que "demander une faveur à» ou "combattre». L'intérêt de telles méthodes est évidemment déterminé par le choix des sources et surtout par le codage opéré sur ces dernières. Si l'on applique des catégories prédéfinies à des sources purement journalistiques, on risque de n'obtenir guère plus qu'une chronologie affinée d'événements bien connus - ou plutôt du regard qu'un organe de presse porte sur eux - et cela au prix de lourds dépouillements ${ }^{59}$. L'énorme base de données élaborée sous la direction de Charles Tilly à partir de 8000 récits d'actions collectives (de la pétition à la violence) ayant eu lieu en GrandeBretagne aux XVIII ${ }^{\mathrm{e}}$ et $\mathrm{XIX}^{\mathrm{e}}$ siècles a permis des traitements plus convaincants, du fait du respect des catégories de l'époque et d'un codage endogène. Ainsi, "Chambre des lords» et "Chambre des communes» n'ont pas été agrégés en «Parlement», car ces "acteurs" n'occupaient pas le même type de position dans les différents réseaux. C'est d'ailleurs à propos de la "parlementarisation» de la

57. L'auteur ne se réfère pas à la théorie des jeux, mais utilise des métaphores renvoyant à des stratégies plus complexes (go, échecs...).

58. J. F. PADGETT, "Organizational genesis, identity and control: the transformation of banking in Renaissance Florence", in James E. RaUCH, Alessandra CASELla (eds), Networks and Markets, New York, Russell Sage, 2001, p. 211-257 (surtout théorique, critiquant les visions simplistes de la modernisation); J. F. PADGETT and Paul D. MCLEAN, «Elite transformation and the rise of economic credit in Renaissance Florence", 2002 (soumis à American fournal of Sociology, téléchargeable sur le web).

59. Malgré l'intérêt de la réflexion méthodologique, les résultats de Roberto FrANzosi, "The return of the actor. interaction among social actors during periods of high mobilization (Italy 1919-1922)", Mobilization, 4/2, automne 1999, p. 131-149 n'apparaissent ainsi guère originaux. Une telle méthode avait déjà été proposée dans les années 1970 (Ronald S. BURT, "Corporate society: a time series analysis of network structure", Social Science Research, 4/4, déc. 1975, p. 271-328), avec l'idée d'un codage totalement positiviste qui ne serait plus guère envisageable de nos jours. L'étude de Peter $\mathrm{S}$. BEARMAN and Kevin D. EverETT, "The structure of social protest, 1961-1983", Social Networks, 15/2, juin 1993, p. 171-200, qui envisage la présence commune dans des manifestations à Washington, souffre des mêmes problèmes de sources et de codage exogène. 
vie politique (le fait que de plus en plus d'acteurs s'adressent au Parlement, ce qui se traduit notamment en termes de centralité) que l'analyse de réseaux, menée avec une périodisation fine, a apporté des résultats convaincants et complémentaires de ceux de sources plus classiques ${ }^{60}$. Employant le même type de méthodes avec le même respect des données, John W. Mohr a, lui, travaillé sur le discours des organisations charitables new-yorkaises vers 1900, mettant en relation les descriptions des actions menées et des groupes sociaux visés avec les évolutions institutionnelles de la période ${ }^{61}$.

\section{De l'importance des liens entre organisations}

Quoique critiques vis-à-vis de l'individualisme méthodologique, les travaux précités prennent le plus souvent l'individu comme unité d'analyse et son engagement comme objet d'étude. Les recherches sur les "mouvements sociaux" ont également exploré la voie d'une étude formalisée des liens entre organisations, permettant de réfléchir à la notion même de "mouvement social", vu comme quelque chose d'agrégé mais de mouvant ${ }^{62}$. Cette démarche peut être vue comme une proposition de réponse aux hypothèses développées en France par Christian Topalov, s'interrogeant sur les notions de "champ» ou de "nébuleuse réformatrice» ${ }^{63}$. Les liens le plus souvent envisagés sont ceux créés par des appartenances multiples de membres, d'autres types de liens (échanges d'informations, de moyens...) laissant moins de traces dans les sources. Formellement, la situation est identique à celle des membres communs à des conseils d'administration d'entreprises : les données sont relativement faciles à formaliser. Interpréter les résultats est moins évident: le partage de membres indique plutôt un lien potentiel que des échanges réels; on peut se demander si la relation est en fait orientée, si une organisation domine l'autre, ou bien si l'une envoie tandis que l'autre reçoit... Il faut dès lors allier à une bonne connaissance du terrain la confrontation de différents traitements possibles (ne compter que les liens multiples, que les liens impliquant des membres de bureaux, prendre en compte la chronologie des adhésions...).

60. Charles TILly, «Parliamentarization of popular contention in Great Britain, 1758-1834», Theory and Society, 26/2-3, avr.-juin 1997, p. 245-273. Pour un prolongement centré sur les différences entre régions: C. Tilly, Lesley J. Wood, "Contentious connections in Great Britain, 1828-34", in M. Diani, D. McAdam (eds), Social Movements..., op. cit., chap. 7. Sur les données, C. Tilly, Popular Contention in Great Britain, 1758-1834, Cambridge, Harvard University Press, 1995.

61. John W. Mohr, Vincent DuQuENNE, "The duality of culture and practice: poverty relief in New York City, 1888-1917", Theory and Society, 26/2-3, avr.-juin 1997, p. 305-356. Sa méthode, l'étude hiérarchique des co-citations, correspond à celle de D. White and H. GILMAN MCCANN, "Cites and fights...", art. cit.

62. Pour un point sur les études "non historiques" de ce type, voir Mario Diani, "Leaders" or brokers? Positions and influence in social movements networks", in M. DiANI, D. McADAM (eds), Social Movements..., op. cit., chap. 5.

63. Christian Topalov, "Le champ réformateur, 1880-1914, un modèle", in C. Topalov (dir.), Laboratoires du nouveau siècle, Paris, Éditions de l'EHESS, 1999, p. 461-474. 
Un article pionnier dans ce domaine porte précisément sur la nébuleuse réformatrice de la fin du XIX ${ }^{\mathrm{e}}$ siècle, dans ses versants féminin et américain ${ }^{64}$. Ce monde qui inclut syndicalistes, antialcooliques ou suffragistes n'est pas facile à cerner, et la position des mouvements féministes en son sein a été débattue de longue date. L'analyse de réseaux permet, sinon de trancher, du moins d'affiner les résultats, notamment en proposant des études de dynamiques, de hiérarchies et d'échanges et non pas simplement la délimitation rigide de frontières entre "mouvements». L'étude des liens entre les 1000 organisations ayant impliqué 200 dirigeantes ou leaders nationales montre la centralité changeante mais forte du mouvement des droits des femmes, seul à unir des «cliques» organisées sur d'autres thèmes. Dans un second article, les mêmes auteurs ont pu comparer des "contextes locaux" différents pour montrer à quel réseau préexistant se confrontent les organisations féministes naissantes et quelles sont leurs stratégies, plus ou moins efficaces, pour se développer. Si les réseaux seuls ne peuvent expliquer échecs ou succès, la description précise des liens permet d'émettre des hypothèses subtiles ${ }^{65}$.

La thèse de Maryjane Osa sur les organisations non communistes en Pologne souligne également qu'il n'existe pas de structure «toujours gagnante" pour un mouvement: l'interprétation de la forme du réseau est relative au contexte ${ }^{66}$. Ainsi, lors des mobilisations de 1954-1959, l'«intermédiarité" apparaît comme une position non de pouvoir, mais de vulnérabilité: l'organisation qui fait le lien entre des groupes très divers est une cible toute désignée pour la répression - ce qui souligne que des acteurs ont une certaine conscience de la forme du réseau. Au contraire, la floraison d'organisations variées et reliées de manière polycentrique à la fin des années 1970, avant même la création de Solidarité, est un des facteurs qui rend la répression plus difficile. Cette recherche, dans laquelle l'analyse de réseaux n'est qu'un pan du raisonnement parmi d'autres, utilise notamment cet outil pour remettre en cause l'idée assez floue d'une "émergence de la société civile", proposant de parler plutôt de sa structuration en un seul réseau assez dense d'organisations.

À partir de ces études s'ouvre une nouvelle direction de recherche, qui vise à mettre en rapports les liens entre individus, entre organisations et entre

64. Naomi Rosenthal, Meryl Fingrutd, Michele Ethier, Roberta Karant, David McDonald, "Social movements and network analysis: a case study of nineteenth-century women's reform in New York State", American fournal of Sociology, 90/5, mars 1985, p. 1022-1054.

65. N. Rosenthal, D. McDonald, M. Ethier, M. Fingrutd, R. Karant, «Structural tensions in the nineteenth-century Women's Movement", Mobilization, 2/1, mars 1997, p. 21-46.

66. Maryjane Osa, Solidarity and Contention. Networks of Polish Opposition, University of Minnesota Press, 2003. Les parties utilisant l'analyse de réseaux avaient été prépubliées: M. OsA, "Mobilizing structures and cycles of protest: post-Stalinist contention in Poland, 1954-1959", Mobilization, 6/2, automne 2001, p. 211-231 et M. OsA, "Networks in opposition: linking organizations through activists in the Polish People's Republic", in M. DiAni, D. McAdAM (eds), Social Movements..., op. cit., chap. 4 (sur les périodes 1966-1970 et 1976-1981). S'agissant d'organisations clandestines, les multi-appartenances ont été reconstituées grâce à une grande variété de sources, policières, privées et orales. 
individus et organisations ${ }^{67}$. Encore peu pratiquée sur des terrains historiques, elle a toutefois été utilisée en France dans la récente thèse de François Denord portant sur les milieux "néo-libéraux", particulièrement composites, de l'entre-deux-guerres ${ }^{68}$. Pour souligner les clivages et les hiérarchies internes à ce mouvement, il utilise à la fois l'analyse factorielle et l'analyse de liens de multi-appartenance, dont il étudie également la signification, entre véritable engagement et simple "multiple prêt" d'un nom prestigieux. L'étude des centralités des individus et des organisations s'ajoute à une caractérisation globale de la structure des liens pour mettre en évidence aussi bien de grands pôles (étatique, patronal ou universitaire) que la place à part d'un individu, qui fait le lien entre organisations tout en jouant un grand rôle dans l'élaboration de la doctrine néo-libérale. Cette recherche montre qu'il est intéressant de lier étude de discours et d'organisations, biographie et analyse de réseaux, et propose des méthodes facilement applicables aux multiples bases prosopographiques constituées en France par l’«histoire des élites».

L'analyse de réseaux ne propose pas, intrinsèquement, une vision irénique du social, qui démontrerait que tout est possible ou refuserait toute notion de structure ou de classe. Ce n'est pas non plus un nouvel objectivisme qui permettrait de cartographier la structure sociale et de prévoir tout comportement individuel. Elle peut présenter le lien à la fois comme une ressource et comme une contrainte, envisager l'individu et ses entourages ou bien les fractures et hiérarchies d'une structure plus large. Une étude historique des réseaux, de leur genèse et de leur évolution est d'ailleurs un bon garde-fou contre tout déterminisme. En outre, une étude systématique des liens peut permettre de conclure à l'inefficacité des réseaux, à la prééminence d'autres phénomènes (de l'ordre de l'attribut, de l'action du marché, de l'institution...): bref, prise comme un outil, l'analyse de réseaux ne conclut pas forcément que "tout est réseau" et peut même permettre d'invalider de tels a priori $^{69}$.

Ce n'est pas non plus un outil miracle pour penser le lien social, du passé ou du présent, mais elle peut aider à conclure sur des hypothèses qui doivent être élaborées au préalable et à partir de sources classiques, simplement envisagées de façon nouvelle. Cette question des sources demeure le principal obstacle à un usage largement répandu de la technique: la première tâche de

67. Proposition dont l'importance a été soulignée par Daniel Roche, "Avant-propos», in P.-Y. BEAUREPAIRE (éd.), La plume et la toile, pouvoirs et réseaux de correspondance dans l'Europe des Lumières, Arras, Presses universitaires d'Artois, 2002, p. 7-24.

68. François DENORD, "Genèse et institutionnalisation du néo-libéralisme en France (années 1930 -années 1950)", thèse de doctorat de sociologie, dir. Rémi Lenoir, EHESS, 2003, chap. VI.

69. Le texte de Georg Fertig (G. FerTig, "Zwischen Xenophobie...", art. cit.) est un bon exemple de cette stratégie de recherche. 
l'historien reste de comprendre "ce que les sources interdisent de faire $»^{70}$. Cela dit, connaître les concepts de l'analyse de réseaux, même sans la pratiquer, peut aider à raisonner en termes moins monolithiques sur les configurations des liens sociaux. Comme pour toute méthode formelle, l'objectif de l'analyse de réseaux reste avant tout de rendre intelligibles des données complexes, à partir de notions discutées collectivement - capital social, cohésion, influence - et des indicateurs correspondants - comme les différentes mesures de la centralité.

L'agenda des sociologues des réseaux, aujourd'hui, comprend au premier chef une étude plus longitudinale et plus dynamique des liens, d'où un intérêt renouvelé de leur part pour les terrains historiques: le moment est donc favorable pour que les historiens entament le dialogue, en insistant sans doute sur leur connaissance des effets de contexte, des sens variables des liens et des mots qui les définissent, ou encore sur la question de la conscience que les acteurs ont de leurs réseaux. Quant aux autres impératifs actuels de la recherche sur les réseaux - attention aux changements d'échelles, aux rapports entre individus et groupes, entre liens et identités - ils sont tout aussi pertinents pour la recherche en histoire sociale et politique. 